\title{
Conditions for Consistency of Equations of Nonlinear Diffusion in Complex Systems with Thermodynamic Constraints
}

\author{
H.P. Sargsyan \\ Institute of Chemical Physics NAS RA, 5/2 Paruir Sevak Str., 0014, Yerevan, Armenia \\ Email: hrachya_sargsyan@mail.ru
}

(Received: September 15, 2021; Revised: October 1, 2021; Accepted: October 7, 2021)

\begin{abstract}
The paper proposes relations between the coefficients of nonlinear diffusion in complex systems within the framework of the mechanism approach of Ya. I. Frenkel for matching equations with thermodynamic constraints. Three conditions for limiting the microdescription of a complex system have been identified the fulfillment of which ensures the thermodynamically correct behavior of the equations of nonlinear multicomponent diffusion.
\end{abstract}

Keywords: nonlinear diffusion, complex system, the stoichiometry of an elementary act, diffusion mechanism, nonlinear diffusion coefficient, thermodynamic constraints

https://doi.org/10.52853/18291171-2021.14.3-154

\section{Introduction}

In early studies, we developed a general formal-kinetic description of nonlinear multicomponent diffusion in complex systems (for example, at different levels of organization of biosystems). We have obtained the general equations of nonlinear diffusion with an arbitrary mechanism of the elementary act of the diffusion jump [1-3]:

$$
\frac{\partial c_{i}}{d t}=\sum_{r} \gamma_{r i} k_{r} \operatorname{div}\left(\sum_{j}\left(\prod_{l} c_{l}^{\alpha_{l}^{I}+\alpha_{r l}^{I I}}\right) \frac{\alpha_{r j}^{I I}}{c_{j}} \vec{\nabla} c_{j}\right)
$$

where $c_{i}=c_{i}(x, t)$ is the functions of coordinates and time - the concentration of coexisting substances of a multicomponent system. It is important to note that, despite the cumbersomeness, the diffusion equations (1) have a rather transparent structure and reflect in sufficient detail the meaning of the mechanism approach of Ya. I. Frenkel [4, 5]. Indeed, in contrast to the generalized equations of multicomponent diffusion based on Fick's diffusion laws [6-8], for which the balance relations and the condition of non-negativity of solutions can be violated, here the dependence of the "diffusion coefficient" on the concentrations of coexisting substances is written explicitly. It should be noted that the diffusion equation in the form (1), which replaces the generalized diffusion equation written in the framework of Fick's second law for diffusion, has one very important advantage: using these equations, numerical models for a computer experiment are easily constructed, with the subsequent determination of the values of the stoichiometric coefficients of the dominant diffusion mechanisms [9-12]. Formally denoting everything that stands in front of $\vec{\nabla} c_{j}$ by $D_{i j}$, the matrix of diffusion:

$$
D_{i j}=\sum_{r} \gamma_{r i} k_{r}\left(\prod_{l} c_{l}^{\alpha_{r l}^{I}+\alpha_{r l}^{I I}}\right) \frac{\alpha_{r j}^{I I}}{c_{j}}
$$

the diffusion equation can be written in the following form:

$$
\frac{\partial c_{i}}{\partial t}=\sum_{j} \operatorname{div}\left(D_{i j} \vec{\nabla} c_{j}\right) \text {. }
$$


The advantages of equations (3) become obvious when diffusion by a specific mechanism is considered. The degree of nonlinearity of the diffusion process depends on the number of components and the properties of a complex system. They are associated with the likelihood of the implementation of a particular mechanism. It is especially important here that the diffusion equations (1) (or (3)) possess the property of preserving the nonnegativity of solutions $c(x)$ under nonnegative initial data and physically reasonable boundary conditions (with a natural constraint on the rate constant of an elementary act of a diffusion jump: $k_{r} \geq 0$ ). The problem of nonnegativeness of solutions of one rather wide class of differential equations was solved by the authors of [13]. The preservation of non-negativity is one of the important properties of equations constructed on the mass action law (MAL).

Equations (1), or (3), are universal in the following sense: first, they describe the process of homogenization - the dissolution of inhomogeneities, similar to linear equations based on Fick's law. Secondly, it is conceivable to realize the conditions under which the main contribution will be made by the mechanisms resulting in the growth of inhomogeneities of the system in the absence of external flows. Mathematically, this means that the corresponding terms of the matrix of diffusion coefficients in (2) have a negative sign. The latter phenomenon is called ascending diffusion.

In the following sections, we will highlight the conditions that provide natural restrictions arising from the laws of thermodynamics.

\section{The condition for the absence of flows in a medium homogeneous in composition}

When formalizing diffusion processes in complex systems, one of the main requirements is to satisfy the condition for the absence of flows in a medium that is homogeneous in composition. According to [1-3], due to diffusion, the flow of matter in a medium, in the cell approximation, is written in the form:

$$
J_{i}=\sum_{r} J_{r i}(I, I I)=-\sum_{r} \gamma_{r i} w_{r}\left(c^{I}, c^{I I}\right),
$$

where $w_{r}\left(c^{I}, c^{I I}\right)$, the speed of an elementary act is set in the form of MAL:

$$
w_{r}\left(c^{I}, c^{I I}\right)=k_{r} \prod_{i}\left(c_{i}^{I}\right)^{\alpha_{r i}^{I}}\left(c_{i}^{I I}\right)^{\alpha_{r i}^{I I}}
$$

$k_{r}$ is the rate constant $\left(k_{r} \geq 0\right)$. It depends both on the kinetic factors of the system under study (the interatomic distances, atomic oscillation frequencies, etc.) and on its thermodynamic properties.

Then the condition for the absence of any flows in a medium homogeneous in composition $\left(c^{I}=c^{I I}\right.$ all components are equal) will look as follows:

$$
\sum_{r} \gamma_{r} w_{r}(c, c)=0
$$

The function $w_{r}(c, c)$, according to (5), is a simple monomial:

$$
w_{r}(c, c)=k_{r} \prod_{i} c_{i}^{\alpha_{r i}^{I}+\alpha_{r i}^{I I}} .
$$

Suppose that among the $n$-dimensional vectors $\alpha_{r}^{I}+\alpha_{r}^{I I}$, having coordinates $\alpha_{r i}^{I}+\alpha_{r i}^{I I}$, there are $q$ different in total. Let us designate them as $y_{1}, \ldots, y_{q}$. For each $p=1, \ldots q$ we put: $Q_{p}$ is the set 
of those numbers $r$ of elementary processes for which $\alpha_{r i}^{I}+\alpha_{r i}^{I I}=y_{p}$. Condition (6) is transformed to the form:

$$
\sum_{r \in Q_{p}} k_{r} \gamma_{r}=0, \quad(p=1, \ldots q)
$$

For example, if we consider diffusion by mechanisms:

$$
n_{r} A_{i}^{I}+m_{r} A_{i}^{I I} \rightarrow p_{r} A_{i}^{I}+q_{r} A_{i}^{I I}
$$

then one set $Q_{p}$ will include those numbers for which the numbers $n_{r}+m_{r}$ have the same meaning (for definiteness, $n_{r}+m_{r}=p$ ). The condition for the absence of flows in a medium homogeneous in composition due to processes (9) can be written in the form:

$$
\sum_{r: n_{r}+m_{r}=p} k_{r}\left(p_{r}-n_{r}\right)=0 .
$$

\section{The principle of detailed equilibrium for ideal systems and its dynamic consequence}

The principle of detailed equilibrium as a consequence of microreversibility was first obtained by Tolman for chemical kinetics (for this, see, for example, [14]). For diffusion, the principle of detailed balance (PDB) expresses the equality of the rate constants of the forward and reverse processes [1-3]:

$$
k_{r}^{+}=k_{r}^{-}\left(=k_{r}\right)
$$

Let (11) is held to be true. Then, the equation of nonlinear multicomponent diffusion (1) can be written in the following form:

$$
\frac{\partial c_{i}}{\partial t}=-\sum_{r} \operatorname{div} \vec{J}_{r i}=\sum_{r} \operatorname{div}\left(\widetilde{w}_{r} \sum_{j} \frac{\gamma_{r i} \gamma_{r j}}{c_{j}} \vec{\nabla} c_{j}\right)
$$

where $\widetilde{w}_{r}=k_{r} \prod_{l} c_{l}^{\alpha_{r l}^{I}+\alpha_{r l}^{I I}}$. The matrix of diffusion coefficients according to (1) and (2) is written as:

$$
D_{i j}(c)=\sum_{r} \widetilde{w_{r}}(c) \frac{\gamma_{r i} \gamma_{r i}}{c_{j}}
$$

It is self-adjoint for the dot product in $R^{n}$ :

$$
<x, y>_{c}=\sum_{i} \frac{x_{i} y_{i}}{c_{i}}
$$

and has non-negative real eigenvalues. If the system of vectors $\left\{\gamma_{r}\right\}$ is complete in $R^{n}$, then the matrix $D_{i j}(c)$ is positively definite for any positive vector $c$. This results in a rapid decay of disturbances with the small wavelength.

Consider Eq. (12) in a bounded domain $U$ of a multicomponent system with a smooth boundary in the absence of flow across the boundary (the vector $\vec{\nabla} c_{i}$ is orthogonal to the normal to 
the boundary $U$ at any of its points). We write the thermodynamic potential $G$ of the region $U$ in the following form:

$$
G(t)=\sum_{i} \int_{U} c_{i}(x, t) \ln c_{i}(x, t) d^{3} x
$$

Let us investigate how $G(t)$ will change with time on solutions $c(x, t)$ of equations (12) under given boundary conditions. By direct computation, we obtain:

$$
\frac{d G}{d t}=\sum_{i} \int_{U}\left(\frac{\partial c_{i}}{\partial t} \ln c_{i}+\frac{\partial c_{i}}{\partial t}\right) d^{3} x
$$

Taking into account (12) and applying Stokes' theorem to (16), we obtain from the condition of the absence of flows through the boundary:

$$
\frac{d G}{d t}=\sum_{i} \int_{U} \ln c_{i} \sum_{r} \operatorname{div}\left(\widetilde{w}_{r} \frac{\gamma_{r i} \gamma_{r j}}{c_{j}} \vec{\nabla} c_{j}\right) d^{3} x
$$

After some transformations and applying Stokes' theorem again, we obtain:

$$
\frac{d G}{d t}=-\sum_{i} \int_{U} \widetilde{w}_{r} \| \vec{\nabla} l n \prod_{i} c_{i}^{\gamma_{r i}}||^{2} d^{3} x,
$$

where \|\|$^{2}$ is the euclidean scalar square vector. Thus, it follows from the right-hand side of (18) that for non-negative initial concentrations, when $\widetilde{w}_{r} \geq 0$, the first derivative of the thermodynamic potential over time is non-positive, that is, the condition is met:

$$
d G / d t \leq 0
$$

It can be seen from (18) that the equality sign in (19) takes place if and only if either one of the factors in $\widetilde{w}_{r}$ is equal to zero, or the gradient:

$$
\vec{\nabla}\left(\prod_{i} c_{i}^{\gamma_{r i}}\right)=0
$$

It is not difficult to verify that, under the diffusion equations (12), the derivative of any function of the form:

$$
G^{*}(t)=\sum_{i} \int_{U} c_{i}(x, t)\left[\ln c_{i}(x, t)+\text { const }_{i}\right] d^{2} x
$$

has the same form as (6). This is because in the course of diffusion, provided there is no flow across the boundary, the values: 


$$
\int_{U} c_{i}(x, t) d^{2} x
$$

They can change either due to a chemical reaction or as a result of the flow of matter across the border.

Thus, we have found that the PDB provides the "thermodynamicity" diffusion (being a sufficient, but certainly not a necessary condition for "thermodynamicity") in a bounded region $U$ with a smooth boundary in the absence of a flow across the boundary (the vector $\vec{\nabla} c_{i}$ is orthogonal to the normal to the boundary $U$ at any point). In other words, the PDB provides the condition for the non-positiveness of the first derivative of the thermodynamic potential of a bounded region $U$ over time: that is the condition hold (19).

\section{The macroscopic consequence of the combined invariance of microdescription}

First, consider the case of a macroscopic consequence of the spatial invariance of the microdescription (by replacing $x$ with $-x$ ). When the space is reflected in the two-cell model, the cells are swapped, which results in the recording of the elementary exchange process [1-3]:

$$
\sum_{i} \alpha_{r i}^{I} A_{i}^{I}+\sum_{i} \alpha_{r i}^{I I} A_{i}^{I I} \rightarrow \sum_{i} \beta_{r i}^{I} A_{i}^{I}+\sum_{i} \beta_{r i}^{I I} A_{i}^{I I}
$$

to change the superscripts of $A_{i}$ (I by II, II by I).

Let us combine the exchange processes (23) in pairs; the mutually inverted connection is under one number. In each pair, we arbitrarily select the initial process, the other will be called the inverted one (this selection is just as arbitrary as the selection of mutually inverse elementary acts of diffusion hopping into the forward and backward directions). According to (5), we write expressions for the rates of direct and inverted processes as:

$$
\begin{aligned}
& w_{r}^{+}=k_{r}^{+} \prod_{i}\left(c_{i}^{I}\right)^{\alpha_{r i}^{I}\left(c_{i}^{I I}\right)^{\alpha_{r i}^{I I}},} \\
& w_{r}^{\prime}=k_{r}^{\prime} \prod_{i}\left(c_{i}^{I I}\right)^{\alpha_{r i}^{I}}\left(c_{i}^{I I}\right)^{\alpha_{r i}^{I I}} .
\end{aligned}
$$

The condition of invariance to spatial reflection means that there exists a set of positive concentrations $c^{*}$ for which the equality is met

$$
w_{r}^{+}\left(c^{*}, c^{*}\right)=w_{r}^{\prime}\left(c^{*}, c^{*}\right)
$$

From this, we easily obtain that:

$$
k_{r}^{+}=k_{r}^{\prime}
$$

The only similarity between (26) and the principle of detailed balancing (11) is that each of these relations implies (8). Both in the presence of detailed equilibrium (11) and the fulfillment of the consequence of the invariance of the microdescription for spatial reflection (26), it is not necessary to impose on the coefficients the additional condition (8) because it is fulfilled automatically.

Combining processes (23) in pairs under one number with inverted to it, the nonlinear diffusion equation (1) can be written in the form: 


$$
\frac{\partial c_{i}}{d t}=\sum_{r} \operatorname{div}\left(\widetilde{w}_{r} \gamma_{r i} \sum_{j} \frac{\alpha_{r j}^{I I}-\alpha_{r j}^{I}}{c_{j}} \vec{\nabla} c_{j}\right)
$$

where, as in (12), $\widetilde{w}_{r}=k_{r} \prod_{l} c_{l}^{\alpha_{r i}^{I}+\alpha_{r i}^{I I}}$.

It seems somewhat unexpected that using (8), any diffusion equation (1) can be written in the form (27). To check this, we group in (1) the terms corresponding to elementary processes with the same sum $\alpha^{I}+\alpha^{I I}$ and substitute instead $\alpha^{I I}$ :

we obtain the equation:

$$
\alpha^{I I}=\frac{\alpha^{I}+\alpha^{I I}}{2}+\frac{\alpha^{I I}-\alpha^{I}}{2}
$$

$$
\frac{\partial c_{i}}{d t}=\sum_{r} \operatorname{div}\left(\left(k_{r}^{+}+k_{r}^{\prime}\right) \gamma_{r i} \prod_{l} c_{l}^{\alpha_{r l}^{I}+\alpha_{r l}^{I I}} \sum_{j} \frac{\alpha_{r j}^{I I}-\alpha_{r j}^{I}}{2 c_{j}} \vec{\nabla} c_{j}\right)
$$

where (8) was used.

By designating $k^{+}+k^{\prime}=\frac{k}{2}$, and isolating $\widetilde{w}_{r}$ similarly to (12), we arrive at equation (27).

Thus, if for the diffusion one uses the mass action law, then relation (26), obtained above from the invariance of the microdescription for spatial reflection, is fulfilled automatically as a consequence of the absence of fluxes in a spatially homogeneous medium. Perhaps, it is this absence of flows, which, within the framework of the stated formalism, is a necessary condition for its applicability, and is the macroscopic expression of the invariance of the microdescription for spatial reflection.

The diffusion coefficient matrix in equation (27) looks like the following:

$$
D_{i j}(c)=\sum_{r} \widetilde{w}_{r}(c) \frac{\gamma_{r i}\left(\alpha_{r j}^{I I}-\alpha_{r j}^{I}\right)}{c_{j}}
$$

The non-negative definiteness of $D_{i j}$ in the form (29) does not become obvious.

Thus, the spatial invariance of the microdescription (its macroscopic consequence) does not ensure the "correct" behavior of solutions.

In other words, for the "thermodynamicity" of diffusion by mechanisms (23), the presence of invariance of the microdescription for the inversion of space (replacing $x$ with $-x$ ) is not a sufficient condition; additional conditions are needed between the coefficients.

Let us discuss the case of invariance of the microdescription concerning the composition of reversals of motion and spatial reflection:

$$
k_{r}^{+}=k_{r}^{-}
$$

Let us combine in pairs, the forward and reverse to the inverted exchange processes under one number. The corresponding diffusion equations from (1) with allowance for (30) will have the form:

$$
\frac{\partial c_{i}}{\partial t}=\operatorname{div}\left(\sum_{r}\left(\prod_{l} c_{l}^{\alpha_{r l}^{I}+\alpha_{r l}^{I I}}\right) k_{r} \gamma_{r i} \sum_{j} \frac{\alpha_{r j}^{I I}+\beta_{r j}^{I}}{c_{j}} \vec{\nabla} c_{j}\right)
$$

Let us transform (31). Suppose that among the vectors with coordinates $\alpha_{r j}^{I}+\alpha_{r j}^{I I}$ there are only $q$ which are different. Let us denote them as $y_{1}, \ldots, y_{q}$. For each $p=1, \ldots, q$ we put: $Q_{p}$ is the 
set of those numbers $r$ for which $\alpha_{r}^{I}+\alpha_{r}^{I I}=y_{p}$. Let us group the terms on the right-hand side of (31) according to this:

$$
\frac{\partial c_{i}}{\partial t}=\sum_{p} \operatorname{div}\left[\left(\prod_{l} c_{l}^{y_{p l}}\right) \times \sum_{r \in Q_{p}} k_{r} \gamma_{r i} \sum_{j} \frac{\alpha_{r j}^{I I}+\beta_{r j}^{I}}{c_{j}} \vec{\nabla} c_{j}\right]
$$

It is easy to see that for every $r \in Q_{p}: \alpha_{r}^{I I}+\beta_{r}^{I}=y_{p}+\gamma_{r}$ takes place. Then the right-hand side of (32) is transformed into the form:

$$
\begin{aligned}
\frac{\partial c_{i}}{\partial t}=\sum_{p} \operatorname{div} & {\left[\left(\prod_{l} c_{l}^{y_{p l}}\right) \sum_{r \in Q_{p}} k_{r} \gamma_{r i} \sum_{j} \frac{y_{p}}{c_{j}} \vec{\nabla} c_{j}\right] } \\
& +\sum_{p} \operatorname{div}\left[\left(\prod_{l} c_{l}^{y_{p l}}\right) \sum_{r \in Q_{p}} k_{r} \gamma_{r i} \sum_{j} \frac{\gamma_{r j}}{c_{j}} \vec{\nabla} c_{j}\right] .
\end{aligned}
$$

Applying the condition of the absence of flows in a medium homogeneous in composition (8), and introducing the notation $\widetilde{w}_{r}$ similarly to (12) (or (27)), we rewrite (33) in the following form:

$$
\frac{\partial c_{i}}{d t}=\sum_{r} \operatorname{div}\left(\widetilde{w}_{r} \sum_{j} \frac{\gamma_{r i} \gamma_{r j}}{c_{j}} \vec{\nabla} c_{j}\right)
$$

which is identical to equation (12) obtained from the principle of detailed balancing.

Thus, the combined invariance of microdescription results in the same dynamic consequences as the principle of detailed balancing. It ensures the "correct" behavior of the solutions. The inhomogeneities dissolve, the thermodynamic potential tends to its equilibrium minimum.

\section{Balance condition and its dynamic consequences}

In the absence of microscopic reversibility, the PDB is replaced by a more general condition of balancing (see [15]) a macroscopic consequence of the microscopic condition of unitarity (preservation of full probability)). Let us formulate a balance condition for the equations of nonlinear diffusion constructed based on the MAL.

Let us use an auxiliary two-cell model. Let $c^{I}=c^{I I}=c^{*}$ be the balance point. Let us write down the thermodynamic potential for the two-cell model in the form:

$$
G=\sum_{i}\left[c_{i}^{I}\left(\ln \left(\frac{c_{i}^{I}}{c_{i}^{*}}\right)-1\right)+c_{i}^{I I}\left(\ln \left(\frac{c_{i}^{I I}}{c_{i}^{*}}\right)-1\right)\right] .
$$

introducing the notation:

$$
\begin{gathered}
\mu_{i}^{I, I I}=\frac{\partial G}{\partial C_{i}^{I, I I}}=\ln \left(\frac{c_{i}^{I, I I}}{c_{i}^{*}}\right) ; \\
\varphi_{r}=k_{r} \prod_{i}\left(c_{i}^{*}\right)^{\alpha_{r i}^{I}+\alpha_{r i}^{I I}}
\end{gathered}
$$


Then (5) can be rewritten as:

$$
w_{r}=\varphi_{r} \exp \left[\left(\alpha_{r}^{I}, \mu^{I}\right)+\left(\alpha_{r}^{I I}, \mu^{I I}\right)\right]
$$

where indicated $\left(\alpha_{r}, \mu\right)=\sum_{i} \alpha_{r i} \mu_{i}$.

By direct calculation from (35), we obtain:

$$
\frac{d G}{d t}=\sum_{r} \varphi_{r}\left[\left(\gamma_{r}, \mu^{I}\right)-\left(\gamma_{r}, \mu^{I I}\right)\right] \times \exp \left[\left(\alpha_{r}^{I}, \mu^{I}\right)+\left(\alpha_{r}^{I I}, \mu^{I I}\right)\right]
$$

We introduce an auxiliary function:

$$
\theta\left(c^{I}, c^{I I}, \lambda\right)=\sum_{r} \varphi_{r} \exp \left[\left((1-\lambda) \alpha_{r}^{I}+\lambda \beta_{r}^{I}, \mu^{I}\right)+\left((1-\lambda) \alpha_{r}^{I I}+\lambda \beta_{r}^{I I}, \mu^{I I}\right)\right]
$$

It is easy to see the identity:

at the point $\lambda=0$.

$$
d G / d t \equiv \partial \theta / \partial \lambda
$$

At each value $c^{I, I I}$ the function $\theta\left(c^{I}, c^{I I}, \lambda\right)$ is a convex function of $\lambda$, therefore, a sufficient condition for the inequality $d G / d t \leq 0$ to hold for all $c^{I, I I}$ ), for any $c^{I, I I}$ is the validity of the inequality,

$$
\theta\left(C^{I}, C^{I I}, 0\right) \geq \theta\left(C^{I}, C^{I I}, 1\right)
$$

The equality is also sufficient

$$
\theta\left(C^{I}, C^{I I}, 0\right)=\theta\left(C^{I}, C^{I I}, 1\right)
$$

The latter is the condition for balance. Let us write it like this:

$$
\sum_{r} \varphi_{r} \exp \left[\left(\alpha_{r}^{I}, \mu^{I}\right)+\left(\alpha_{r}^{I I}, \mu^{I I}\right)\right]=\sum_{r} \varphi_{r} \exp \left[\left(\beta_{r}^{I}, \mu^{I}\right)+\left(\beta_{r}^{I I}, \mu^{I I}\right)\right]
$$

Let among all pairs of vectors $\left(\alpha_{r}^{I}, \alpha_{r}^{I I}\right),\left(\beta_{r}^{I}, \beta_{r}^{I I}\right)$ be total $l$ different pairs. Let us denote them as $\left(x^{1}, z^{1}\right),\left(x^{2}, z^{2}\right), \ldots,\left(x^{l}, z^{l}\right)$. The set of those numbers $r$ of elementary exchange processes for which $\alpha_{r}^{I}=x^{i}, \alpha_{r}^{I I}=z^{i}$, we denote by $R_{i+}$, and those numbers $r$ for which $\beta_{r}^{I}=x^{i}, \beta_{r}^{I I}=z^{i}$ we denote as $R_{i-}$. Then, taking into account $\alpha_{r i}^{I}+\alpha_{r i}^{I I}=\beta_{r i}^{I}+\beta_{r i}^{I I}$ and (36), the condition of balance (43) can be written in the form:

$$
\sum_{r \in R_{i+}} k_{r}=\sum_{r \in R_{i-}} k_{r}
$$

Here we show that the balance condition for nonlinear diffusion written in the form (44), gives the same dynamic consequences as the principle of detailed balancing and replaces it in the absence of microreversibility.

To do this, we use the expression for the equation of nonlinear multicomponent diffusion based on the MAL in the following form [1-4]:

$$
\frac{\partial c_{i}(x, t)}{\partial t}=-\sum_{r} \operatorname{div} \overrightarrow{J_{r l}}=\sum_{r} \gamma_{r i} \operatorname{div}\left(\left.\sum_{j} \frac{\partial w_{r}\left(c^{I}, c^{I I}\right)}{\partial c_{j}^{I I}}\right|_{c^{I}=c^{I I}=c(x)} \vec{\nabla} c_{j}\right)
$$


and substitute the velocity function in it in the form (37). As a result, we obtain the diffusion equation instead of (45):

$$
\frac{\partial c_{i}}{d t}=\sum_{r} \gamma_{r i} \operatorname{div}\left(\widetilde{w}_{r} \sum_{j} \alpha_{r j}^{I I} \vec{\nabla} \mu_{j}\right),
$$

where $\widetilde{w}_{r}=k_{r} \prod_{l} c_{i}^{\alpha_{r i}^{I}+\alpha_{r i}^{I I}}, \quad \mu_{j}=\ln c_{j}$.

Let us define the thermodynamic potential of a bounded region $U$ with a smooth boundary in the absence of a flow across the boundary in the following form:

$$
G=\sum_{i} \int_{U} c_{i}(x, t) \ln c_{i}(x, t) d^{3} x
$$

By direct calculation, we obtain for $\frac{d G}{d t}$ from (47),

$$
\frac{d G}{d t}=-\sum_{i, j, r} \int_{U} \widetilde{w}_{r} \gamma_{r i} \alpha_{r j}^{I I}\left(\vec{\nabla} \mu_{i}, \vec{\nabla} \mu_{j}\right) d^{3} x,
$$

(where (46) is taken into account and the Stokes' theorem is used). For $d G / d t$ to be nonpositive on any positive solution (46), it is necessary and sufficient that the quadratic form

$$
Q_{c}(x)=\sum_{i, j, r} \widetilde{w}_{r} \gamma_{r i} \alpha_{r j}^{I I} x_{i} x_{j}\left(=\sum_{i, j} q_{i j} x_{i} x_{j}\right)
$$

was nonnegatively defined: $Q_{c}(x) \geq 0$ for any $x$ and positive $c$.

Let us verify that the balance condition (44) is sufficient for the nonnegativity of the righthand side of (49) for all positive $c$.

Taking into account (8), the matrix $q_{i j}$ from (49) we transform like this:

$$
q_{i j}=\sum_{r} w_{r}^{o} k_{r} \gamma_{r i} \alpha_{r j}^{I I}=-\sum_{r} w_{r}^{o} k_{r} \gamma_{r i} \alpha_{r j}^{I},
$$

where $w_{r}^{o}=\prod_{l} c_{l}^{\alpha_{r l}^{I}+\alpha_{r l}^{I I}}$.

From the condition of conservation of the amount of matter in the absence of chemical transformations: $\alpha_{r i}^{I}+\alpha_{r i}^{I I}=\beta_{r i}^{I}+\beta_{r i}^{I I}$ it follows that $w_{r}^{o}$ is the same value for mutually inverse processes. Then we also have (taking into account):

$$
\begin{aligned}
& \quad q_{i j}=\sum_{r} w_{r}^{o} k_{r} \beta_{r i}^{I} \alpha_{r j}^{I I}-\sum_{r} w_{r}^{o} k_{r} \alpha_{r i}^{I} \alpha_{r j}^{I I}=\sum_{r} w_{r}^{o} k_{r} \beta_{r i}^{I} \alpha_{r j}^{I I}-\sum_{r} w_{r}^{o} k_{r} \beta_{r i}^{I} \beta_{r j}^{I I}= \\
& =\sum_{r} w_{r}^{o} k_{r} \gamma_{r j} \beta_{r i}^{I}
\end{aligned}
$$


It is easy to see that by replacing $q_{i j}$ in (49) by $\left(q_{i j}+q_{j i}\right) / 2$ the quadratic form of $Q_{c}$ does not change. Then, using expression (50) for $q_{i j}$, and (51) for $q_{j i}$, we have

$$
q_{i j}+q_{j i}=\sum_{r} w_{r}^{o} k_{r}\left(-\gamma_{r i} \alpha_{r j}^{I}+\gamma_{r i} \beta_{r j}^{I}\right)=\sum_{r} w_{r}^{o} k_{r} \gamma_{r i} \gamma_{r j} .
$$

Finally, for a quadratic form $Q_{c}(x)$ we obtain:

$$
Q_{c}=\frac{1}{2} \sum_{r} w_{r}^{o} k_{r}\left(\gamma_{r}, x\right)^{2} \geq 0
$$

Thus, instead of (48), for $d G / d t$ we obtain expression of the form:

$$
\frac{d G}{d t}=-\frac{1}{2} \sum_{r} \int_{U} \widetilde{w}_{r}\left\|\vec{\nabla} l n \prod_{i} c_{i}^{\gamma_{r i}}\right\|^{2} d^{3} x \leq 0 .
$$

The nonnegative definiteness of the quadratic form $Q_{c}(x)$ also implies the nonnegativeness of the eigenvalues of the matrix of diffusion coefficients:

$$
D_{i j}(c)=\sum_{r} \widetilde{w}_{r}(c) \frac{\gamma_{r i} \alpha_{r j}^{I I}}{c_{j}}
$$

Indeed, we have that there exists a scalar product for each $c$ :

$$
\left\langle\frac{x}{y}\right\rangle_{c}=\sum_{i} \frac{x_{i} y_{i}}{c_{i}}
$$

such that the quadratic form $\langle x / D x\rangle$ can be written as:

$$
\left\langle\frac{x}{D x}\right\rangle=\sum_{i, j} q_{i j} \frac{x_{i}}{c_{i}} \frac{y_{i}}{c_{i}}=Q_{c}(y)
$$

where $y_{i}=x_{i} / c_{i}$. Then, we have from (53):

$$
\langle x / D x\rangle \geq 0
$$

which means the nonnegativity of the eigenvalues of the matrix of diffusion coefficients (55).

If the exchange mechanism is such that each formal sum found in its record

$$
X=\sum_{i} \alpha_{i}^{I} A_{i}^{I}+\sum_{i} \alpha_{i}^{I I} A_{i}^{I I}
$$

exactly once stands on the left in the stoichiometric equation $(X \rightarrow \cdots)$ and exactly one time on the right $(\cdots \rightarrow X)$, then, the PDB follows (11) from the balance condition (44). 


\section{Conclusions}

Thus, within the framework of the theory of nonlinear diffusion in multicomponent systems, where we can apply the MAL developed by us in our early works [1-3, 16], an important addition has been made: for the equations of nonlinear multicomponent diffusion, three matching conditions with thermodynamic constraints are formulated. The first and most important requirement for any theory of diffusion is to ensure the absence of any fluxes in a compositionally homogeneous isolated system. In the second section of this work, a fairly transparent relationship is obtained between the quantities characterizing the elementary act of diffusion transfer in the two-cell approximation. According to the general principles of thermodynamics, only such processes can take place in a closed system that results in an increase of entropy, or a decrease in the Gibbs thermodynamic potential. Here we have identified three conditions, the fulfillment of which ensures the thermodynamically correct behavior of the system due to diffusion processes. The first of these conditions is a macroscopic consequence of the invariance of the microdescription of an isolated system to the time-reversal fulfillment of microreversibility. This is the fulfillment of equality (11), the PDB for diffusion. The second condition is the combined invariance of the microdescription to the reversal of time and the reflection of space, the fulfillment of condition (30), the rate constants of the elementary act of diffusion of the direct process, and the process inverted to the reverse are equal. And, finally, we have shown that in the absence of microreversibility and/or invariance of the microdescription to the reflection of space, there is a more general condition of balance (44), which is a macroscopic consequence of the microscopic condition of unitarity (the preservation of full probability, for this you can see the work [17]).

In conclusion, I would like to express my deep gratitude to Professor Aleksandr Nikolaevich Gorban for invaluable assistance in researching the development of formalism for a quasi-chemical description of nonlinear multicomponent diffusion.

\section{References}

[1] A.N. Gorban, H.P. Sargsyan, H.A. Wahab, Armenian Journal of Physics 12 (2019) 45.

[2] H.P. Sargsyan, A.H. Sargsyan, A.M. Danielyan, Journal of Contemporary Physics (Armenian Academy of Sciences) 54 (2019) 326.

[3] H.P. Sargsyan, Armenian Journal of Physics 13 (2020) 7.

[4] J. Frenkel, Zeitschrift für Physik 26 (1924) 117.

[5] J. Frenkel, Zeitschrift für Physik 35, (1925) 652.

[6] M.A. Krishtal, A.I. Volkov, Multicomponent diffusion in metals (Moscow: Metallurgy, 1985).

[7] B.Ya. Lyubov, Diffusion processes in inhomogeneous solid media (Moscow: Nauka, 1981).

[8] L.G., Voroshin, B.M. Khusid. Diffusion mass transfer in multicomponent systems (Minsk: Science and Technology, 1979).

[9] C. Tuijn, Defect and Diffusion Forum 11 (1997) 143.

[10] D.T. Gillespie, Annu. Rev. Phys. Chem. 58 (2007) 35.

[11] G.M. Poletaev, I.V. Zorya, D.V. Novoselova, M.D. Starostenkov. Intern. Journal of Materials Research 108 (2017) 785.

[12] S. Mahmoud, M. Trochet, O.A. Restrepo, N. Mousseau, Acta Materialia 144 (2018) 679.

[13] A.I. Volpert, V.S. Posvyanski, Chem. Phys. 3 (1984) 1200.

[14] A.N. Gorban, V.I. Bykov, G.S. Yablonskii, Essays on chemical relaxation (Novosibirsk: Nauka, 1986).

[15] A.N. Gorban, Equilibrium encircling. Equatins of Chemical Kinetics and Their Thermodynamics Analysis (Novosibirsk: Nauka, 1984).

[16] A.N. Gorban, H.P. Sargsyan, Kinetics and Catalysis 27 (1986) 527.

[17] E.C.G. Stueckelberg, Helv. Phys. Acta 25 (1952) 577. 